\title{
A Topographic Instructive Signal Guides the Adjustment of the Auditory Space Map in the Optic Tectum
}

\author{
Peter S. Hyde and Eric I. Knudsen \\ Department of Neurobiology, Stanford University School of Medicine, Stanford, California 94305
}

\begin{abstract}
Maps of auditory space in the midbrain of the barn owl (Tyto alba) are calibrated by visual experience. When owls are raised wearing prismatic spectacles that displace the visual field in azimuth, the auditory receptive fields of neurons in the optic tectum shift to compensate for the optical displacement of the visual field. This shift results primarily from a shift in the tuning of tectal neurons for interaural time difference. The visually based instructive signal that guides this plasticity could be based on a topographic, point-by-point comparison between auditory and visual space maps or on a foveation-dependent visual assessment of the accuracy of auditory orienting responses. To distinguish between these two possibilities, we subjected owls to optical conditions that differed in the center of gaze and the visual periphery. A topographic signal would
\end{abstract}

The experience-dependent alignment of the auditory space map with the visual space map in the optic tectum of the barn owl (Tyto alba) is a well studied example of supervised learning, in which one neural network controls plasticity in another (Knudsen, 1994). Precise calibration of the auditory map underlies the owl's ability to orient its gaze accurately toward auditory stimuli (Brainard and Knudsen, 1998). Adjustments of the auditory space map result from changes in the tuning of tectal neurons for interaural time difference (ITD) and interaural level difference (ILD), the dominant cues for sound source azimuth and elevation, respectively, in barn owls (Olsen et al., 1989).

The calibration of the auditory space map is guided by visual experience. When juvenile barn owls are subjected chronically to prismatic spectacles that displace the visual field horizontally, neurons in the optic tectum gradually alter their tuning for ITD to compensate for the optical displacement caused by the prisms (Brainard and Knudsen, 1993). In contrast, in adult owls that have been raised normally, ITD tuning changes little in response to equivalent prism experience. However, in adult owls that acquired a learned ITD map as juveniles, ITD tuning in the tectum can shift back and forth between normal and learned tuning, depending on recent visual experience (Knudsen, 1998).

The instructive signal that guides adaptive changes in ITD tuning could be generated in two different ways (Knudsen, 1994).

\footnotetext{
Received April 17, 2001; revised July 13, 2001; accepted Aug. 16, 2001.

This work was supported by grants from the March of Dimes and from National Institute on Deafness and Other Communication Disorders Grant 5 R01 DC00155-20 to E.I.K. and a Lieberman Fellowship from Stanford University School of Medicine to P.S.H. We thank Yoram Gutfreund, Brie Linkenhoker, and Wemin Zheng for helpful comments on this manuscript.

Correspondence should be addressed to Peter S. Hyde, Department of Neurobiology, Stanford University School of Medicine, Fairchild Building, Room D255, Stanford, CA 94305. E-mail: psh@leland.stanford.edu.

Copyright (C) 2001 Society for Neuroscience $\quad 0270-6474 / 01 / 218586-08 \$ 15.00 / 0$
}

cause the portions of the space map representing the central and peripheral regions of visual space to adjust differently, according to the optical conditions that exist in each region. In contrast, a foveation-based signal would cause both portions of the map to adjust similarly, according to the optical conditions that exist at the center of gaze. In six of seven experiments, adaptive changes were as predicted by a topographic instructive signal. Although the results do not rule out the possible contribution of a foveation-based signal, they demonstrate that a topographic instructive signal is, indeed, involved in the calibration of the auditory space map in the barn owl optic tectum.

Key words: supervised learning; superior colliculus; multimodal maps; instructive signal; plasticity; barn owl

A topographic, template-based signal could be derived from a topographic representation of visual space. Such a signal could guide changes in the auditory space map by reinforcing auditory connections that contribute to activity patterns that match that of the visually based template and weakening auditory connections that do not. Alternatively, an instructive signal could be derived from a visual assessment of the accuracy of auditory orienting responses to bimodal (auditory-visual) stimuli. Such a signal could guide changes in the auditory space map by strengthening auditory connections that contribute to orienting responses that cause the stimulus to fall in the owl's center of gaze and weakening auditory connections that do not. We refer to such a non-topographic signal as a foveation-based instructive signal. Computational modeling has shown that either type of signal can account for the effects of prism experience on the auditory space map (Gelfand et al., 1989; Rucci et al., 1997).

In this study, we subjected the center of gaze and a region of the peripheral visual field to different optical conditions. Under the conditions we imposed, a topographic, template-based signal and a foveation-based signal would lead to different patterns of adjustment in the portions of the tectum representing the two regions of space: a template-based signal would result in different amounts of adjustment in the two portions of the tectum, whereas a foveation-based signal would result in equal adjustments in both portions of the tectum. The results demonstrate that different portions of the auditory space map can, and usually do, adjust differentially, indicating that a topographic instructive signal contributes to the alignment of the auditory space map in the barn owl optic tectum.

\section{MATERIALS AND METHODS}

Five barn owls (Tyto alba) were studied. The owls lived in flight cages with other owls and were cared for in accordance with the Stanford University Institutional Animal Care and Use Committee and the $\mathrm{Na}$ - 
tional Institutes of Health Guide for the Care and Use of Laboratory Animals.

\section{Surgical procedures}

The owls were prepared for experiments when they reached $\sim 60 \mathrm{~d}$ of age. Twelve to $24 \mathrm{hr}$ before surgery, they were removed to individual cages and deprived of food. The owls were anesthetized with halothane $(1.5 \%)$ mixed with nitrous oxide and oxygen (45:55) and wrapped in a soft leather jacket. A stainless steel plate for fixing the head during electrophysiological experiments was cemented to the base of the skull, and a stainless steel spectacle frame mount was cemented to the front of the skull. Craniotomies were opened bilaterally over the optic tecta, filled with antibiotic ointment, and sealed with dental acrylic. All incisions were infused with lidocaine hydrochloride. After surgery, the owls were given intramuscular injections of fluids and monitored in individual cages until they fully recovered.

\section{Visual experience}

To determine the nature of the visually based instructive signal, we exposed the center of gaze and a peripheral region of the visual field to different optical conditions and tested whether the portions of the tectal auditory space map representing these two regions of the visual field adjusted similarly or differently. We defined the center of gaze as the region of the visual field along the horizon from left $5^{\circ}$ to right $5^{\circ}$ and the periphery as the region from $15^{\circ}$ to $30^{\circ}$ in azimuth to one side. This particular peripheral region of the visual field was chosen for analysis because, across experiments, the effect of the spectacles on this portion of the field was uniform and free of the edge effects discussed below.

The plasticity of the auditory space map varies across individuals (Brainard and Knudsen, 1998). To assess the capacity for plasticity in each individual and in each portion of the map, we began the experiment by subjecting each owl to a horizontal displacement of the visual field. In all five owls, Fresnel prismatic lenses (VisionCare/3M, St. Paul, MN), mounted in lightweight aluminum frames, were attached to the spectacle frame mounts at $\sim 60 \mathrm{~d}$ of age. Three owls were fitted with prisms that displaced the visual field $23^{\circ}$ to the right, one owl was fitted with prisms that displaced the visual field $23^{\circ}$ to the left, and one owl was fitted with prisms that displaced the visual field $17^{\circ}$ to the left. The prisms were centered on the visual axes and displaced the central $80^{\circ}$ of the visual field. Owls wore these spectacles for $6-8$ weeks, during which time the auditory space map changed adaptively according to the prismatic displacement of the visual field. The average magnitude of the adjustment in the portions of the tectum representing the center of gaze and the periphery defined the "initial mean shift in best ITD" for each portion of the tectum in each owl. The initial mean shift in best ITD was used for normalizing observed shifts in ITD tuning measured in subsequent experiments (see below).

The experiments described here measured the effects of partial occlusion of the visual field on either the recovery of normal or the reacquisition of learned ITD tuning. The initial adjustment of auditory tuning in response to prismatic displacement of the visual field has been shown to involve the generation of new anatomical circuitry (DeBello et al., 2001). The contribution of anatomical changes to subsequent adjustments of the map has not been measured, but is likely to be much less. Therefore, the initial map adjustment changes the properties of the network in a special way. There is no reason to think, however, that the initial adjustment should change the nature of the instructive signal that guides the changes in auditory tuning.

After the initial experience with prismatic displacement of the visual field, opaque plastic occluders were mounted in front of both eyes so that the same restricted region of the visual field was blocked binocularly. The region of the visual field occluded by the spectacles was assessed in the dark by viewing the retinas ophthalmoscopically using a calibrated perimetry system. The occluders created three distinct regions of the visual field. One region of the visual field, from which the retinas could not be sighted with the pupils fully dilated, was totally obstructed all of the time. The neighboring region, $\sim 10^{\circ}$ in width, was intermittently obstructed depending on pupillary dilation (range, $7-10^{\circ}$ ) and eye position (maximum displacement $<3^{\circ}$ from the zero position; du Lac and Knudsen, 1990). We refer to this region of the visual field as the intermittently interrupted region. The remainder of the visual field was not affected by the occluders. This region of the visual field was normal (clear lenses) when testing for recovery of normal ITD tuning, or optically displaced by prisms when testing for the reacquisition of learned ITD tuning.

After the initial prismatic displacement of the visual field, all five owls were fitted with spectacles that completely occluded one side of the visual field peripheral to $15^{\circ}$ in azimuth and allowed normal vision at the center of gaze. This condition was maintained until the mean best ITD measured in the portion of the tectum representing the center of gaze returned to within $15 \mu \mathrm{sec}$ of normal, a period that ranged from $\sim 2-5$ weeks. Two of these owls were then subjected to a second experiment. Both owls were exposed to 1-3 weeks of normal visual experience, during which the entire auditory space map returned approximately to normal. One owl was then fitted with spectacles that completely obstructed the right visual field beyond $5^{\circ}$, intermittently interrupted the center of gaze, and displaced the rest of the left visual field by $23^{\circ}$ to the left. The second owl was fitted with spectacles that occluded the right visual field peripheral to $15^{\circ}$ in azimuth and displaced the center of gaze, by $17^{\circ}$ to the left.

During the course of these experiments, spectacles were checked and cleaned three times per week. Owls wearing spectacles that occluded part of the visual field (like owls wearing standard prismatic spectacles) appeared to fly, feed, and interact normally.

\section{Electrophysiology}

The effect of visual experience on ITD tuning in the optic tectum was assessed electrophysiologically. Twelve to $24 \mathrm{hr}$ before electrophysiological experiments, owls were removed to individual cages and deprived of food. On the day of the experiment, they were anesthetized as described above and wrapped in a soft leather jacket. Craniotomies were opened, and the exposed, intact dura mater was covered with antibiotic ointment. The owl was suspended in a prone position in a sound isolation chamber. The head was attached to a stereotaxic frame using the plate cemented to the base of the skull and aligned in the apparatus using retinal landmarks, as described previously (Knudsen, 1982). During the course of the experiment, owls were maintained on nitrous oxide and oxygen (45:55). If the owl became restless, $1 \%$ halothane was added to the mixture until the owl became quiet again, at which point the halothane was turned off.

Electrode penetrations were made vertically, in the plane perpendicular to the owl's visual axes, using insulated tungsten microelectrodes $(0.1-2 \mathrm{M} \Omega$ at $1 \mathrm{kHz})$. Multiunit responses were isolated using a level discriminator, and spike timing was stored on a Silicon Graphics Indy computer. Multiunit sites recorded in the same penetration were separated by a minimum of $400 \mu \mathrm{m}$.

Visual receptive fields were measured by projecting positive or negative contrast visual stimuli onto a tangent screen in front of the owl while monitoring responses with an audio monitor. Visual receptive fields were first measured without the spectacles in place. The effect of the spectacles on the visual receptive field of a site was verified by replacing the spectacles and measuring visual responses again.

Auditory stimuli were presented dichotically via matched earphones (Knowles, ED-1941 with attached baffles, BF-1743) centered in the external ear canals, $\sim 5 \mathrm{~mm}$ from the eardrums. The stimuli were digitally generated, broadband $(1-12 \mathrm{kHz})$ noise bursts, $50 \mathrm{msec}$ in duration (rise-fall time $=0 \mathrm{msec}$ ), presented at $15-30 \mathrm{~dB}$ above threshold. Tuning for ITD was assessed by presenting a series of noise bursts with ITD varied in a random, interleaved manner while ILD was held at the optimal value for the site. Responses were defined as the number of spikes recorded in the $100 \mathrm{msec}$ after stimulus presentation minus the baseline number of spikes recorded in the $100 \mathrm{msec}$ before stimulus presentation. The best ITD of a tectal site was defined as the midpoint of the range of ITDs for which the response exceeded $50 \%$ of the maximum response.

The effect of visual experience on ITD tuning was determined by comparing the best ITD measured at each site with the best ITD predicted based on the normal, reliable relationship between best ITD and visual receptive field azimuth. Because visual receptive field location is not altered by prism experience (Brainard and Knudsen, 1993), we were able to use the azimuth of the visual receptive field of each site (measured without prisms) to calculate its predicted normal best ITD and the shift in best ITD according to the following formulas (Brainard and Knudsen, 1993):

predicted best ITD $($ in $\mu \mathrm{sec})=2.5 *$ visual RF azimuth (in degrees)

$$
\text { shift in best ITD }=\text { predicted best ITD }- \text { measured best ITD. }
$$

Positive values of shift represent differences from normal ITD tuning in the direction predicted by the optical displacement of the prisms (the adaptive direction), and negative values of shift represent differences in the opposite, nonadaptive direction. For each experiment, we calculated 
Table 1. Changes in ITD tuning with center of gaze normal and periphery occluded

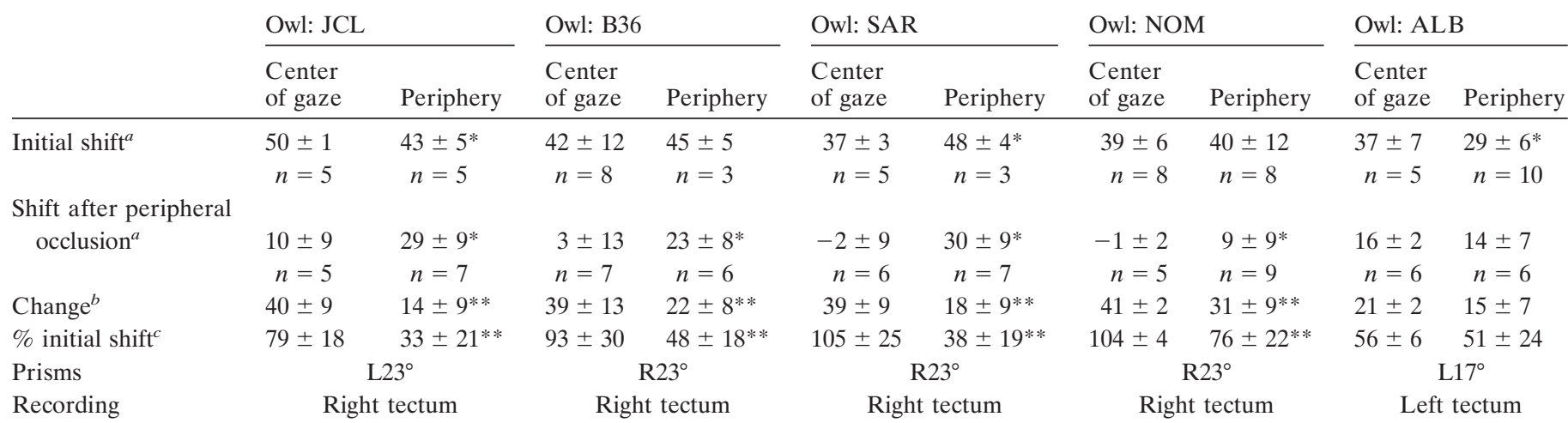

\footnotetext{
${ }^{a}$ Mean best ITD relative to predicted normal (in microseconds).

${ }^{b}$ Change $=$ initial shift - shift after peripheral occlusion (in microseconds).

$c \%$ Initial shift $=($ change/initial shift $) * 100$.

* Mean best ITD relative to normal in the periphery differs from that at the center of gaze $(p<0.05$; two-tailed $t$ test).

**Changes in the occluded periphery were smaller than those at the non-occluded center of gaze $(p<0.05$; two-tailed $t$ test).
}

the mean shift in best ITD in the portions of the tectum representing the center of gaze and the periphery, respectively. Data from sites with visual receptive fields outside these regions of the visual field are shown in Figures 1-4 but were not included in the calculation of mean shifts.

The change in mean best ITD that occurred in each portion of the tectum in each owl during partial occlusion of the visual field was calculated as: mean shift before occlusion - mean shift after occlusion. We normalized these changes in mean best ITD to the potential shift in best ITD for each portion of the tectum. In experiments examining the effect of partial occlusion on the recovery of normal ITD tuning: potential shift in best ITD = mean shift after initial prisms. In experiments examining the reacquisition of shifted ITD tuning: potential shift in best ITD $=$ mean shift after initial prisms - mean shift after normal visual experience.

\section{Predictions}

Topographic, template-based instructive signal. A topographic template signal would cause different auditory adjustments in the representations of the occluded and non-occluded portions of the space map according to the optical conditions that exist in each portion of the visual field. Specifically, such a signal would instruct large adaptive shifts at sites with non-occluded visual receptive fields and would provide no instruction at sites with occluded visual receptive fields. In addition, at the resulting boundaries between differentially shifted portions of the space map, the transition in ITD tuning should be graded, reflecting the spatial resolution of the instructive signal and the degree of lateral, excitatory interactions within the map: the lower the spatial resolution and the greater the lateral spread of excitation, the more gradual the transition in auditory tuning between the shifted and unshifted portions of the map.

Foveation-based instructive signal. A foveation-based signal would instruct equal auditory adjustments in the representations of the occluded and non-occluded portions of the space map under the conditions we used. We specifically avoided conditions where the center of gaze and the periphery are subjected to different, unobstructed visual input (such as a prismatic displacement of the center of gaze and normal vision in the periphery), which could lead to regional differences in auditory adjustment across the map if the visuomotor system made region-specific adjustments to enable accurate foveation of stimuli in both zones (Rucci et al., 1997).

A foveation-based signal could cause regional differences in adaptive adjustment across the map under our conditions if different portions of the auditory space map were more plastic than others. However, previous research has demonstrated that this is not the case (Brainard and Knudsen, 1993; Gold and Knudsen, 2000). Moreover, the capacity for plasticity in each portion of the map was documented for each bird in the first part of this experiment (Table 1, top row) and was controlled in the analysis.

Nonvisual influences. In addition to the effects of a visually based instructive signal, nonvisual influences will also contribute to changes in auditory tuning. Nonvisual spatial information could be provided by the somatosensory and proprioceptive systems and by spatial information that is intrinsic to the auditory system (Mogdans and Knudsen, 1992). There is also an innate preference for neurons in the space map to be tuned to normal values of ITD (Knudsen et al., 1991; Brainard and Knudsen, 1998). These nonvisual influences will always tend to drive auditory tuning toward normal. Their effects will be most apparent in experiments that begin with a shifted auditory space map, tending to shift auditory tuning in both occluded and nonoccluded portions of the map back toward normal. Note that these nonvisual influences cannot account for, or contribute to, shifts in auditory tuning away from normal.

\section{RESULTS}

\section{Capacity for plasticity}

The initial prism experience, lasting 6-8 weeks, established the capacity for plasticity (the initial mean shift in best ITD) in the portions of the optic tectum representing the center of gaze and the periphery for each owl (Table 1, top row). In two owls (owls NOM and B36), the mean shift in best ITD did not differ between the portions of the tectum representing the center of gaze and periphery, respectively ( $p>0.05$; two-tailed $t$ test). In two other owls (owls JCL and ALB), ITD tuning in the portion of the tectum representing center of gaze shifted further, by 7 and 8 $\mu$ sec, respectively, and in one owl (owl SAR), ITD tuning in the portion of the tectum representing the periphery shifted further, by $11 \mu \mathrm{sec}(p<0.05$; two-tailed $t$ test).

\section{Effect of peripheral visual field occlusion on reacquisition of a normal ITD map}

After we had assessed the initial shift of the map, we replaced the prisms of all five owls with spectacles that restored normal vision to a region of the visual field including the center of gaze and obstructed vision in a peripheral region of the visual field. The changes in ITD tuning that would be expected in the corresponding portions of the tectum differ depending on whether the instructive signal is topographic or foveation-based (Materials and Methods). In brief, a topographic signal would instruct adjustments toward normal only in the portion of the tectum representing the nonoccluded center of gaze, whereas a foveationbased signal would instruct changes toward normal in both portions of the tectum. Nonvisual influences will also tend to drive tuning in both portions of the map back toward normal.

For all five owls, subsequent experience with partially occluded, normal vision caused ITD tuning to shift toward normal in both portions of the tectum representing the nonoccluded 

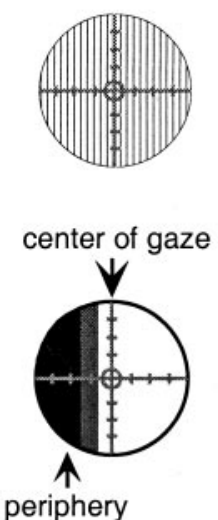

Center of Gaze

A
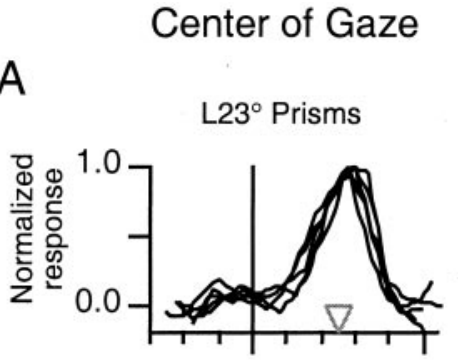

D

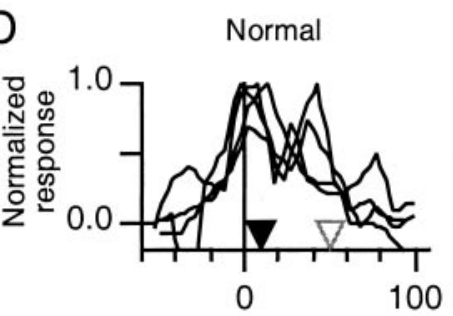

Periphery B

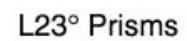

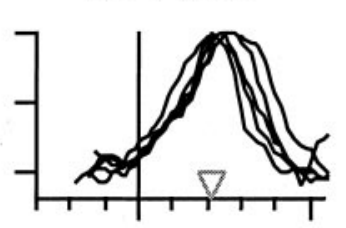

E Occluded

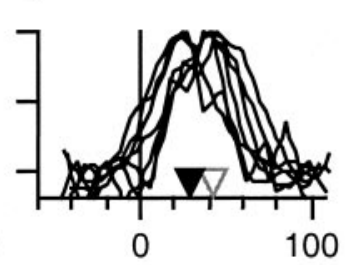

0

100
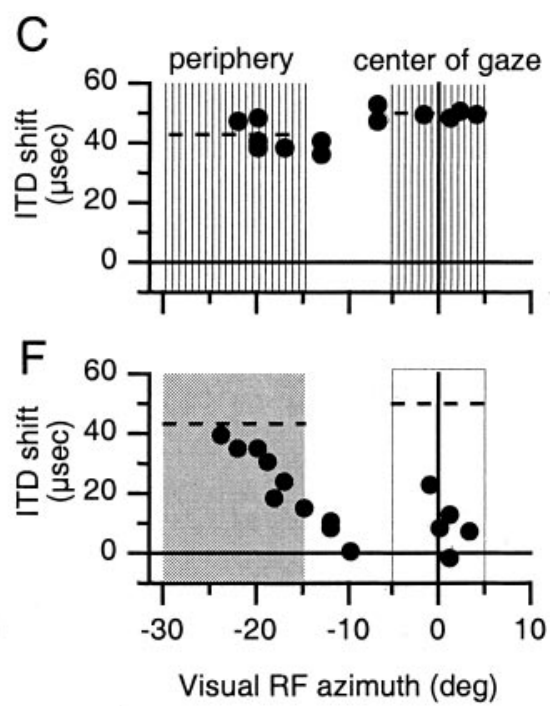

Figure 1. The effect of normal vision at the center of gaze and occlusion of the periphery on ITD tuning in the optic tectum. Circles on the left represent the visual field (central circle $=$ visual axis, hatch marks $=10^{\circ}$ increments) and indicate the nature of the visual experience before the experiments shown. Vertical hatching indicates a region of the visual field displaced by prisms, lack of shading indicates a normal, nondisplaced region of the visual field, gray shading indicates an intermittently interrupted region of the field (see Materials and Methods), and black shading represents a region of the field binocularly obstructed by occluders. $A, B$, ITD tuning curves at sites with visual receptive fields in the center of gaze $(A)$ and the periphery $(B)$ measured in owl JCL after 7 weeks of experience with L23 ${ }^{\circ}$ displacement of the visual field. Open triangles indicate the initial mean shift in best ITD. $C$, Shift in best ITD relative to predicted normal plotted versus visual receptive field azimuth for all tectal sites recorded in owl JCL after the initial prismatic displacement of the visual field. Vertical hatching indicates that the regions of the visual field defined as the center of gaze (L5 to R $\left.5^{\circ}\right)$ and the periphery $\left(\mathrm{L} 15^{\circ}\right.$ to $\left.\mathrm{L} 30^{\circ}\right)$ had been prismatically displaced. Dashed lines indicate the initial mean shift in best ITD in the corresponding portions of the tectum. $D, E$, ITD tuning curves at sites with visual receptive fields in the center of gaze $(D)$ and the periphery $(E)$ measured after $11 \mathrm{~d}$ of normal vision at the center of gaze and occlusion of the periphery beyond $\mathrm{L}^{\circ} 5^{\circ}$. For each portion of the tectum, black triangles indicate the mean shift in best ITD, and white triangles indicate the mean initial shift in best ITD (from above). F, Shift in best ITD relative to predicted normal plotted versus visual receptive field azimuth for all sites recorded after normal vision at the center of gaze and occlusion of the periphery. Dashed lines indicate the initial mean shift in best ITD measured in the corresponding portions of the tectum (from above). The shaded box indicates the region of the visual field defined as the periphery, which had been occluded, and the open box indicates the region defined as the center of gaze, which had experienced normal vision.

center of gaze and the occluded periphery, respectively $(p<$ 0.01 ; two-tailed $t$ test) (Table 1 ). In four of the five owls, the change in ITD tuning in the portion of the tectum representing the nonoccluded center of gaze was greater than that in the portion of the tectum representing the occluded periphery $(p<$ 0.05; two-tailed $t$ test) (Table 1). The results from one of these four owls (owl JCL) are shown in Figure 1. The initial mean shift in best ITD for sites with visual receptive fields in the center of gaze was $50 \pm 1 \mu \mathrm{sec}$ and that for sites with visual receptive fields in the left periphery was $43 \pm 5 \mu \mathrm{sec}$ (Fig. $1 A-C$ ); these values represent the potential ITD shift that could occur in these regions of the tectum after the restoration of normal vision. After $11 \mathrm{~d}$ of experience with normal vision at the center of gaze and occluded vision in the periphery, the mean shift in best ITD for sites with visual receptive fields in the center of gaze had changed from $50 \pm 1$ to $10 \pm 9 \mu \mathrm{sec}(p<0.01$; two-tailed $t$ test $)$, and that for sites with visual receptive fields in the periphery had changed from $43 \pm 5$ to $29 \pm 9 \mu \mathrm{sec}(p<0.01$; two-tailed $t$ test) (Fig. $1 D-F)$. The magnitude of these changes, both in terms of absolute change and percentage of the initial shift, was significantly larger in the portion of the tectum representing the nonoccluded center of gaze than in the portion representing the occluded periphery ( $p<0.01$; two-tailed $t$ test) (Table 1$)$. As expected, there was a gradual change in the degree of ITD tuning shift at the boundary between the shifted and nonshifted portions of the space map (predictions in Materials and Methods). Similar results from three other owls are shown in Figure 2.
In one owl (owl ALB), ITD tuning shifted equally in both portions of the tectum (Table 1). This owl was the only owl that experienced a $17^{\circ}$ (as opposed to a $23^{\circ}$ ) displacement of the visual field and it had the smallest initial shifts in both portions of the tectum before fitting the occluders.

\section{Effect of interruption of the center of gaze on the reacquisition of a learned ITD map}

We subjected the owl used in the experiment described in Figure 1 (owl JCL) to a second experiment. First, the owl was given 1 week of normal visual experience, during which the ITD map returned to normal (Fig. $3 A-C$ ). Then, we occluded the peripheral visual field beyond $\mathrm{R}^{\circ}$ (rather than beyond $\mathrm{L}^{\circ} 5^{\circ}$, as in the previous experiment) and prismatically displaced the rest of the visual field (Fig. 3). This caused the intermittently interrupted portion of the visual field to be coextensive with the center of gaze (see Materials and Methods); we did not completely obstruct the center of gaze, because we were concerned that complete obstruction would jeopardize the owl's ability to survive in the flight room with other owls. Under these conditions, a topographic signal would instruct a shift in auditory tuning at sites with visual receptive fields in the optically displaced periphery, whereas instruction would be intermittently interrupted at the center of gaze. A foveation-based signal would be intermittently interrupted for both portions of the tectum. Nonvisual influences would tend to prevent shifts away from normal across the tectum (see Materials and Methods). 


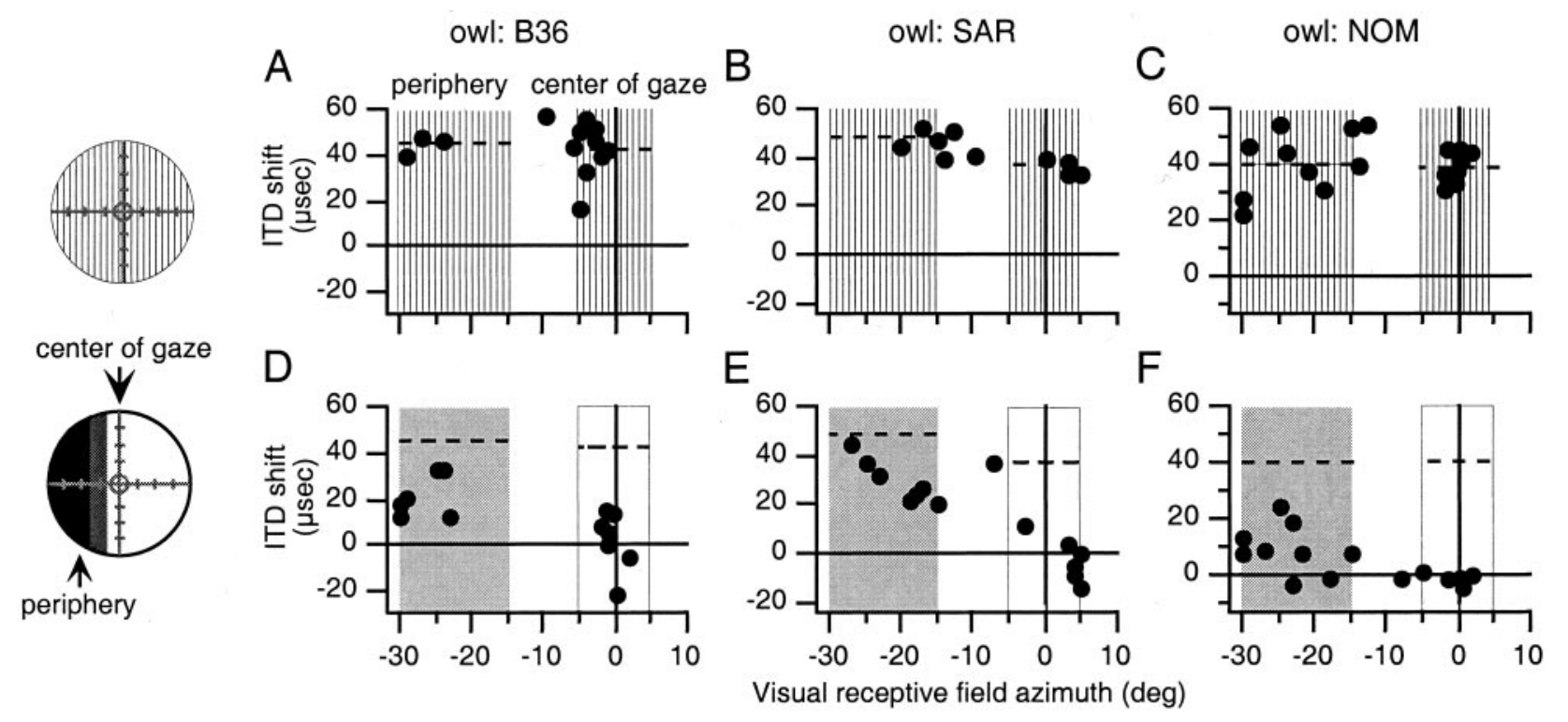

Figure 2. The effect of normal vision at the center of gaze and occlusion of the periphery on best ITDs in the optic tectum. Circles on the left represent the visual field and indicate the nature of the visual experience before the experiments shown (conventions as in Fig. 1). $A-C$, Shift in best ITD relative to predicted normal plotted versus visual receptive field azimuth for all sites recorded after the initial experience with prismatic displacement of the visual field in owls B36, SAR, and NOM, respectively. Conventions are the same as in Figure $1 C$. $D-F$, Shift in best ITD plotted versus visual receptive field azimuth for all sites recorded after normal vision at the center of gaze and occlusion of the periphery in owls B36 (35 d), SAR (14 d), and NOM $(14 \mathrm{~d})$, respectively. Conventions are the same as in Figure $1 F$.
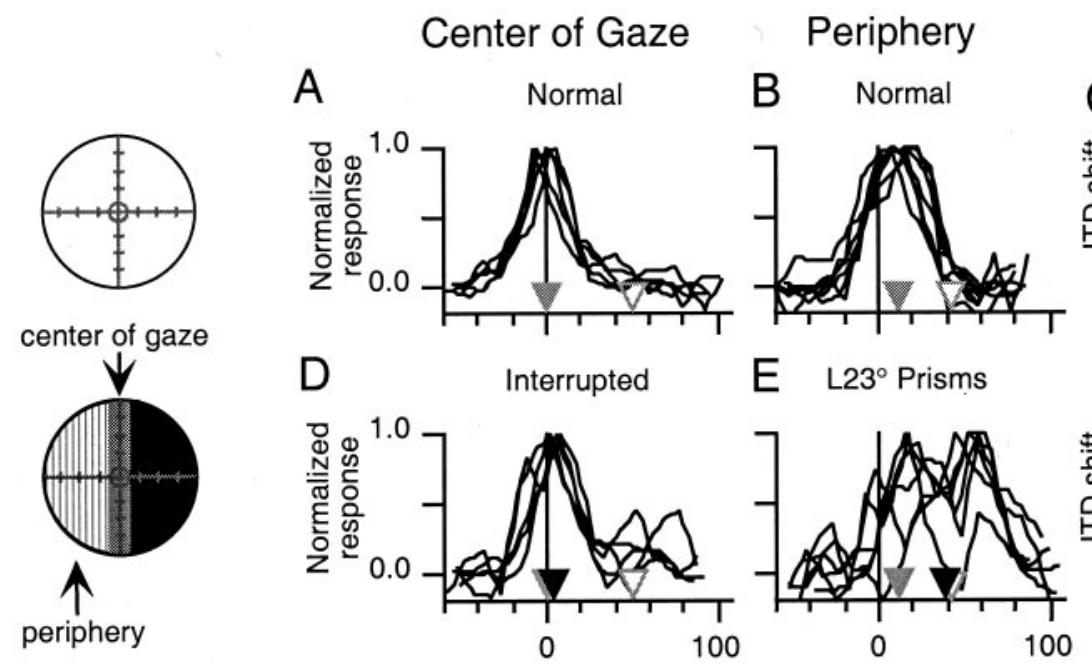

ITD re: predicted normal $(\mu \mathrm{sec})$
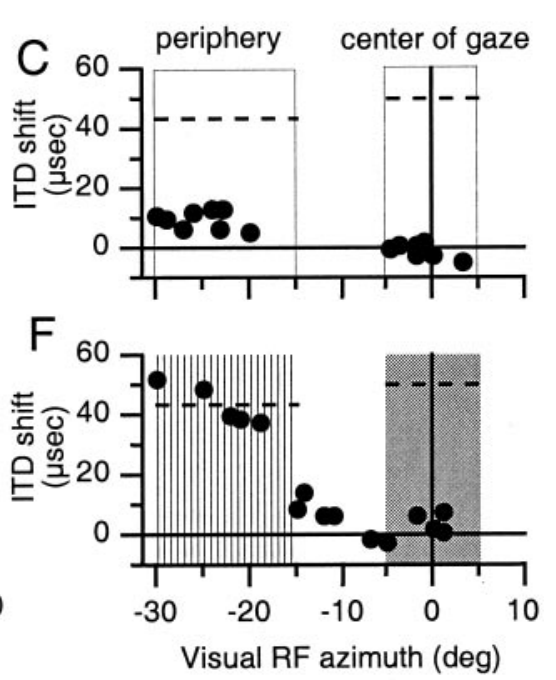

Figure 3. Effect of intermittent interruption of the center of gaze and prismatic displacement of the periphery. Circles on the left represent the visual field and indicate the nature of the visual experience before the experiments shown (conventions as in Fig. 1). $A, B$, ITD tuning curves of sites with visual receptive fields in the center of gaze $(A)$ and the periphery $(B)$ measured in owl JCL after normal visual experience. Gray triangles indicate the mean shift in best ITD, and white triangles indicate the initial mean shift in best ITD in the corresponding portions of the tectum (from Fig. $1 A, B$ ). $C$, Shift in best ITD plotted versus visual receptive field azimuth for all tectal sites recorded in owl JCL after normal visual experience. Open boxes indicate normal vision in the regions of the visual field defined as the center of gaze and the periphery. Dashed lines indicate the initial mean shift in best ITD in the corresponding portions of the tectum (from Fig. $1 C)$. $D, E$, ITD tuning curves of sites with visual receptive fields in the center of gaze $(D)$ and the periphery $(E)$ measured after $21 \mathrm{~d}$ of experience with intermittent interruption of the center of gaze and prismatic displacement of the periphery. For each portion of the tectum, black triangles indicate the mean shift in best ITD after this manipulation of the visual field, gray triangles indicate the mean shift in best ITD after normal visual experience, and white triangles indicate the initial mean shifts in best ITD from Figure 1C. F, Shift in best ITD relative to predicted normal plotted versus visual receptive field azimuth for all sites recorded after partial occlusion of the center of gaze and optical displacement of the periphery. Vertical hatching indicates the region of the visual field defined as the periphery, which had been displaced prismatically, and shading indicates the region of the visual field defined as the center of gaze, which had been interrupted intermittently. Dashed lines indicate the initial mean shifts in best ITD in the corresponding portions of the tectum from Figure $1 C$. 
Center of Gaze

A

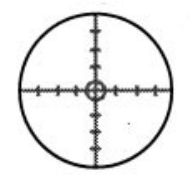

center of gaze

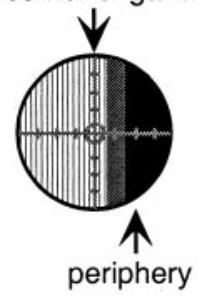

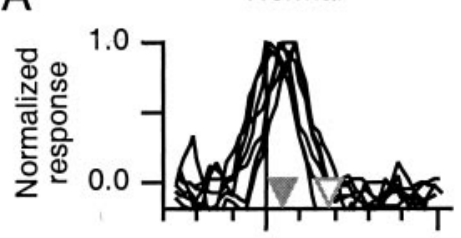

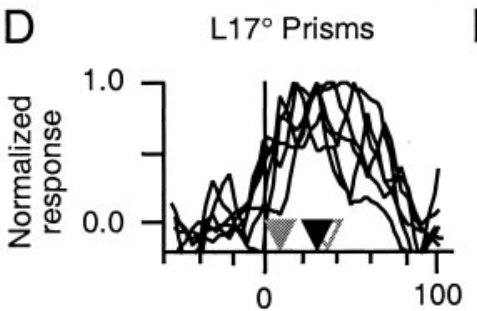

ITD re: predicted normal $(\mu \mathrm{sec})$
Periphery

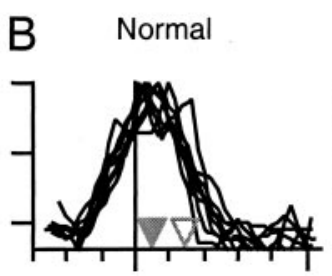

E Occluded

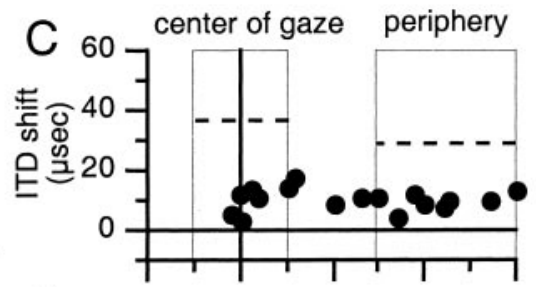

$\mathrm{F} 60$

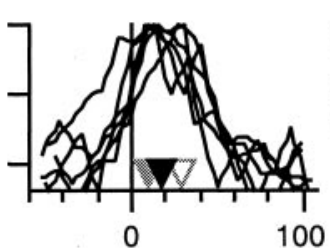

100

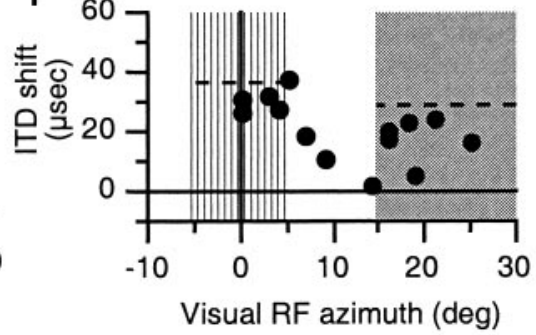

Figure 4. Effect of prismatic displacement of the center of gaze and occlusion of the periphery. Circles on the left represent the visual field and indicate the nature of the visual experience before the experiments shown (conventions as in Fig. 1). $A, B$, ITD tuning curves of sites with visual receptive fields in the center of gaze $(A)$ and the periphery $(B)$ measured in owl ALB after normal visual experience. Conventions are the same as in Figure $3, A$ and $B$; initial mean shifts (white triangles) are from Table 1 (owl ALB). $C$, Shift in best ITD relative to predicted normal plotted versus visual receptive field azimuth for all tectal sites recorded after normal visual experience. Open boxes indicate normal vision in the regions of the visual field defined as the periphery and the center of gaze. Dashed lines indicate the initial mean shift in best ITD in the corresponding portions of the tectum (from Table 1 , owl ALB). $D, E$, ITD tuning curves of sites with visual receptive fields in the center of gaze $(D)$ and the periphery $(E)$ measured after $42 \mathrm{~d}$ of prismatic displacement of the center of gaze and occlusion of the periphery. Conventions are the same as in Figure $3, C$ and $D$. F, Shift in best ITD plotted versus visual receptive field azimuth for all sites recorded after prismatic displacement of the center of gaze and occlusion of the periphery. Vertical hatching indicates the region of the visual field defined as the center of gaze, which had been displaced, and shading indicates the region of the visual field defined as the periphery, which had been occluded.

The results of this experiment are summarized in Figure 3. After 1 week of normal visual experience, the mean shift in best ITD was $-1 \pm 3 \mu \mathrm{sec}$ in the portion of the tectum representing the center of gaze and $11 \pm 3 \mu \mathrm{sec}$ in the portion of the optic tectum representing the left periphery (Fig. $3 A-C$ ). For this experiment, the potential shift in best ITD (initial mean shift mean shift after normal experience) was $51 \mu \mathrm{sec}$ in portion of the tectum representing the center of gaze and $32 \mu \mathrm{sec}$ in the portion of the tectum representing the periphery. Interruption of the center of gaze prevented changes in ITD tuning from occurring in the portion of the tectum representing the center of gaze (Fig. 3D) $(p>0.05$; two-tailed $t$ test $)$. At the same time, prismatic displacement of the periphery caused a dramatic shift in mean best ITD, from $11 \pm 3$ to $38 \pm 15 \mu \mathrm{sec}$, in the portion of the tectum representing the optically displaced periphery (Fig. $3 E$ ) ( $p<0.01$; two-tailed $t$ test). This change was $84 \%$ of the potential shift in best ITD for this portion of the tectum (Fig. $3 F$ ). There was little or no change in ITD tuning in the portion of the tectum representing the displaced region of the visual field between $\mathrm{L} 5^{\circ}$ and $\mathrm{L} 15^{\circ}$.

In the two experiments performed with this owl (Figs. 1, 3), ITD tuning in the portion of the optic tectum representing the left periphery changed dramatically when the left peripheral region of the visual field was optically displaced (Fig. 3) and changed little when the left peripheral region of the visual field was occluded (Fig. 1). This demonstrates, in a single owl, that adjustments in ITD tuning depended specifically on the visual conditions existing in the corresponding regions of space and are not the result of differences in the capacity for plasticity in the two portions of the tectum.

\section{Effect of peripheral occlusion on reacquisition of a learned ITD map}

We subjected the owl that did not show differential changes in the first experiment (owl ALB) to a second experiment. First, the owl was given 3 weeks of normal visual experience, during which the ITD map shifted substantially toward normal (Fig. $4 A-C$ ). Then, we displaced the center of gaze $17^{\circ}$ to the left and occluded the periphery beyond $\mathrm{R} 15^{\circ}$. Under these conditions, a topographic signal would instruct sites with visual receptive fields in the displaced center of gaze to adjust ITD tuning in the adaptive direction, whereas it would provide no instructive signal in the occluded periphery. A foveation-based signal would instruct similar shifts in ITD tuning in both portions of the tectum, according to the optical displacement at the center of gaze. Nonvisual influences would tend to prevent shifts away from normal across the tectum (see Materials and Methods).

The results of this experiment are summarized in Figure 4. After $17 \mathrm{~d}$ of normal experience, the mean shift in best ITD was $9 \pm 5 \mu \mathrm{sec}$ for sites with visual receptive fields in the center of gaze and $10 \pm 3 \mu \mathrm{sec}$ for sites with visual receptive fields in the periphery (Fig. $4 A-C$ ). The potential shift in best ITD (initial mean shift - mean shift after normal experience) was $28 \mu \mathrm{sec}$ in portion of the tectum representing the center of gaze and $19 \mu \mathrm{sec}$ in the portion of the tectum representing the right periphery (Fig. $4 C$ ). After 7 weeks of experience with a prismatically displaced center of gaze and occluded periphery, the mean shift in best ITD had changed from $9 \pm 5$ to $31 \pm 4 \mu \mathrm{sec}(p<0.01$; two-tailed $t$ test) in the portion of the tectum representing the center of gaze and from $10 \pm 3$ to $18 \pm 7 \mu \sec (p<0.05$; two-tailed $t$ test) in the portion of the tectum representing the occluded periphery (Fig. $4 D-F)$. The change was larger in the portion of the tectum 

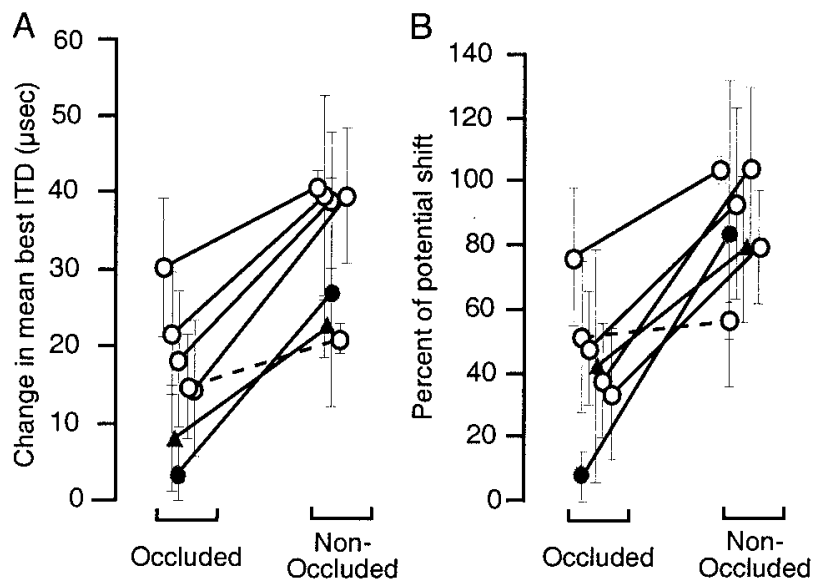

Figure 5. Summary of changes in ITD tuning in the portions of the optic tectum representing occluded and nonoccluded regions of the visual field. $A$, Absolute changes in mean shift in best ITD in the occluded and nonoccluded portions of the tectum. Positive values indicate changes in the direction predicted by the visual conditions present in the nonoccluded region of the visual field. Lines connect points measured in the same owl (solid lines: two-tailed $t$ test, $p<0.05$; dashed lines: two-tailed $t$ test, $p>0.05)$. Error bars indicate the SD of the shift in best ITD. Open circles represent data from the five experiments in which the center of gaze was normal and the periphery was occluded (Figs. 1, 2, Table 1). Filled circles represent data from the experiment in which the center of gaze was interrupted and the periphery was optically displaced (Fig. 3). Filled triangles represent data from the experiment in which the center of gaze was optically displaced and the periphery was occluded (Fig. 4). B, Changes in ITD tuning in the occluded and nonoccluded portions of the optic tectum expressed as a percentage of potential shift in best ITD (see Materials and Methods). Conventions are the same as in $A$.

representing the prismatically displaced center of gaze than in the portion of the tectum representing the occluded periphery, both in terms of absolute change and in terms of percentage of potential shift ( $p<0.05$; two-tailed $t$ test).

\section{Summary}

In six of seven experiments, we observed changes in ITD tuning in the predicted direction in both portions of the tectum, representing the occluded and the nonoccluded regions of the visual field, respectively. In the remaining experiment, significant changes occurred only in the portion of the tectum representing the nonoccluded visual field (Fig. 3). In five of the six experiments, in which changes occurred in both portions of the tectum, both the absolute and normalized changes in the nonoccluded (either optically displaced or normal) portion of the tectum were larger than those in the occluded portion $(p<0.05$; two tailed $t$ test) (Fig. 5).

\section{DISCUSSION}

These experiments used partial occlusion of the visual field to examine the nature of the visually based instructive signal that guides the alignment of the auditory space map in the barn owl optic tectum. The results demonstrate that a topographic, template-based signal contributes to the alignment of the auditory space map. This is consistent with results in the ferret indicating that topographic visual input to the superficial layers of the superior colliculus is required for the normal development of the auditory space map (King et al., 1998). Our results do not rule out a possible, additional contribution of a foveation-based instructive signal, but they can be explained in the absence of such a signal. First, we discuss the results that specifically support the action of a topographic, instructive signal. Then we discuss other influences that may contribute to the alignment of the auditory space map. Finally, we consider two potential anatomical sources for the topographic instructive signal.

\section{Evidence for a topographic, template-based instructive signal}

A template-based instructive signal would align each portion of the auditory space map with a topographic representation of visual space in a point-by-point manner. If such a signal were to contribute to experience-dependent changes in ITD tuning in the tectum, interruption or elimination of visual input from a specific region of space should interfere with the ability of the corresponding portion of the tectal auditory space map to adjust its auditory tuning. Consistent with this prediction, visual occlusion diminished the amount of auditory adjustment in the portion of the tectum representing a visually occluded region of space in six of seven experiments (Fig. 5). In one case, the adjustments that occurred in both regions of the tectum were not different (Table 1, owl ALB; Fig. 5). However, this exception can be accounted for by the additional action of other influences on the auditory map, discussed below. In contrast, the differential adjustments, which were larger always in the non-occluded portion of the map, can only be accounted for (under the conditions used in this experiment) by an instructive signal that acts as a topographic template.

\section{Other influences on auditory space map alignment}

Although visual occlusion differentially diminished auditory plasticity in the corresponding portions of the optic tectum in most cases, it did not prevent it completely. In six of seven experiments, significant changes in the predicted direction occurred in the portions of the tectum where vision was obstructed (Table 1, Fig. $5)$. These changes can be explained by the action of one, or several, additional influences on auditory spatial tuning in the tectum.

These changes could be caused by a second, nontopographic instructive signal. This second instructive signal could be a foveation-based signal. Importantly, the changes observed in the occluded portion of the tectum were significantly smaller than those observed in the nonoccluded portion in five of six experiments, suggesting that such a signal would have to act in concert with the topographic signal to achieve complete adaptive change. However, in the experiment shown in Figure 3, in which large adaptive changes occurred in the representation of the periphery in the absence of changes at the interrupted center of gaze, the changes must have been accomplished without the aid of such a foveation-based signal.

Nonvisual influences provided by other sensory modalities, such as audition, somatic sensation, and proprioception (Mogdans and Knudsen, 1992), as well as an innate preference for normal tuning in the space map (Knudsen et al., 1991; Brainard and Knudsen, 1998) can account for the changes in auditory tuning that were observed in the occluded portion of the tectum in those cases in which tuning changed from a shifted state toward normal (Figs. 1, 2, Table 1). In the occluded portion of the tectum that lacked visual instruction from the topographic signal, these influences would tend to drive auditory tuning back toward normal, as was observed. In addition, the innate preference for normal tuning likely contributed to the lack of adaptive shift in the displaced portion of the tectum between $\mathrm{L}^{\circ}$ and $\mathrm{L} 15^{\circ}$ in the experiment shown in Figure 3.

An uninstructed change toward normal tuning could also have 
occurred in owls that began with shifted maps of ITD (Figs. 1, 2, Table 1) as a result of disinhibition of normal circuitry. It has been shown that, at sites expressing shifted ITD tuning, the circuitry underlying normal ITD tuning is still present, but responses to it are suppressed by inhibition (Zheng and Knudsen, 1999). Release from this inhibition causes ITD tuning to shift back toward normal. Thus, it is possible that the change toward normal tuning, observed in the occluded portions of the tectum in these cases, was attributable to the disinhibition of normal circuitry in the absence of active instructive input.

Another, nonexclusive possibility that can account for the shifts in tuning in the occluded portion of the tectum in all of the experiments is the influence of lateral interactions within the auditory space map. There is an extensive network of lateral connections within the optic tectum (Hunt and Brecha, 1984). One role of these connections may be to help maintain the continuity of the auditory space map (Knudsen and Brainard, 1991). Lateral excitatory connections from shifted portions of the space map could contribute to shifts in tuning at sites with visual receptive fields in the occluded region of the visual field. Instructed changes may begin in the center of the nonoccluded portion of the tectum and progress outward toward the borders with the occluded portions of the tectum. Lateral excitatory connections from the shifted portion of the map could pull the ITD tuning of sites in the neighboring, uninstructed, occluded portions of the tectum in the same direction. Conversely, lateral excitatory connections in uninstructed portions of the tectum could act to slow changes in adjacent, instructed portions of the tectum by the same mechanism. Such an effect of lateral interactions is suggested by the graded variations in ITD tuning shifts that were observed in several cases at the boundary between shifted and unshifted portions of the map (Figs. $1 F, 3 F, 4 F$ ).

\section{Possible sources of a topographic instructive signal}

These experiments demonstrate that a topographic instructive signal contributes to the alignment of the auditory space map in the optic tectum. Such a signal could be generated by using the topographic visual input to the tectum as a template to which the auditory space map is aligned (Gelfand et al., 1989). Interestingly, however, the initial site of experience-dependent plasticity in the midbrain auditory localization pathway is not in the optic tectum, but in the external nucleus of the inferior colliculus (ICX), the nucleus before the optic tectum (Brainard and Knudsen, 1993). Visual input to the ICX has been sought, but never found. How, then, might a topographic instructive signal reach the ICX?

The ICX receives inputs from two structures capable of providing instructive information. A portion of the forebrain called the auditory archistriatum (AAr) also sends a projection to the ICX. The AAr contains a representation of auditory space (Cohen et al., 1998) that is adaptively altered by prism experience (Miller and Knudsen, 1999). Although the representation of auditory space in the AAr is not topographic, the signal it provides to the tectum could be topographically organized. In addition, the optic tectum sends a point-to-point, feedback projection to the ICX that could provide a topographic instructive signal (Hyde and Knudsen, 2000; Luksch et al., 2000). Most of the tectal neurons that project to the ICX have dendrites in the visual and auditory recipient layers of the tectum. Experiments are being conducted to determine whether either or both of these structures are the source of the topographic instructive signal that is predicted by the results reported in this study.

\section{REFERENCES}

Brainard MS, Knudsen EI (1993) Experience-dependent plasticity in the inferior colliculus: a site for visual calibration of the neural representation of auditory space in the barn owl. J Neurosci 13:4589-4608.

Brainard MS, Knudsen EI (1998) Sensitive periods for visual calibration of the auditory space map in the barn owl optic tectum. J Neurosci 18:3929-3942.

Cohen YE, Miller GL, Knudsen EI (1998) Forebrain pathway for auditory space processing in the barn owl. J Neurophysiol 79:891-902.

DeBello WM, Feldman DE, Knudsen EI (2001) Adaptive axonal remodeling in the midbrain auditory space map. J Neurosci 21:3161-3174.

du Lac S, Knudsen EI (1990) Neural maps of head movement vector and speed in the optic tectum of the barn owl. J Neurophysiol 63:131-149.

Gelfand JJ, Pearson JC, Spence CD, Sullivan WE (1989) Multisensor integration in biological systems. In: Proceedings, IEEE International Symposium on Intelligent Control 1988, pp 147-153.

Gold JI, Knudsen EI (2000) Abnormal auditory experience induces frequency-specific adjustments in unit tuning for binaural localization cues in the optic tectum of juvenile owls. J Neurosci 20:862-877.

Hunt SP, Brecha N (1984) The avian optic tectum: a synthesis of morphology and biochemistry. In: Comparative neurology of the optic tectum (Vanegas H, ed), pp 619-648. New York: Plenum.

Hyde PS, Knudsen EI (2000) Topographic projection from the optic tectum to the auditory space map in the inferior colliculus of the barn owl. J Comp Neurol 421:146-160.

King AJ, Schnupp JW, Thompson ID (1998) Signals from the superficial layers of the superior colliculus enable the development of the auditory space map in the deeper layers. J Neurosci 18:9394-9408.

Knudsen EI (1982) Auditory and visual maps of space in the optic tectum of the owl. J Neurosci 2:1177-1194.

Knudsen EI (1994) Supervised learning in the brain. J Neurosci 14:3985-3997.

Knudsen EI (1998) Capacity for plasticity in the adult owl auditory system expanded by juvenile experience. Science 279:1531-1533.

Knudsen EI, Brainard MS (1991) Visual instruction of the neural map of auditory space in the developing optic tectum. Science 253:85-87.

Knudsen EI, Esterly SD, DuLac S (1991) Stretched and upside-down maps of auditory space in the optic tectum of blind-reared owls; acoustic basis and behavioral correlates. J Neurosci 11:1727-1747.

Luksch H, Gauger B, Wagner H (2000) A candidate pathway for a visual instructional signal to the barn owl's auditory system. J Neurosci 20:RC70.

Miller GL, Knudsen EI (1999) Early visual experience shapes the representation of auditory space in the forebrain gaze fields of the barn owl. J Neurosci 19:2326-2336.

Mogdans J, Knudsen EI (1992) Vision-independent adjustment of unit tuning to sound localization cues in response to monaural occlusion in developing owl optic tectum. J Neurosci 12:3485-3493.

Olsen JF, Knudsen EI, Esterly SD (1989) Neural maps of interaural time and intensity differences in the optic tectum of the barn owl. J Neurosci 9:2591-2605.

Rucci M, Tononi G, Edelman GM (1997) Registration of neural maps through value-dependent learning: modeling the alignment of auditory and visual maps in the barn owl's optic tectum. J Neurosci 17:334-352

Zheng W, Knudsen EI (1999) Functional selection of adaptive auditory space map by GABAA-mediated inhibition. Science 284:962-965. 